\title{
Multilevel dual approach for pricing American style derivatives ${ }^{1}$
}

\author{
Denis Belomestny ${ }^{2}$, John Schoenmakers ${ }^{3}$, Fabian Dickmann²
}

October 12,2012

\begin{abstract}
In this article we propose a novel approach to reduce the computational complexity of the dual method for pricing American options. We consider a sequence of martingales that converges to a given target martingale and decompose the original dual representation into a sum of representations that correspond to different levels of approximation to the target martingale. By next replacing in each representation true conditional expectations with their Monte Carlo estimates, we arrive at what one may call a multilevel dual Monte Carlo algorithm. The analysis of this algorithm reveals that the computational complexity of getting the corresponding target upper bound, due to the target martingale, can be significantly reduced. In particular, it turns out that using our new approach, we may construct a multilevel version of the well-known nested Monte Carlo algorithm of Andersen and Broadie (2004) that is, regarding complexity, virtually equivalent to a non-nested algorithm. The performance of this multilevel algorithm is illustrated by a numerical example.
\end{abstract}

Keywords: optimal stopping, dual approach, multilevel Monte Carlo

AMS Subject Classification: 62L15, 65C05, 91B28

\section{Introduction}

Efficient methods for pricing high-dimensional American options have been a challenge for decades. While for a low or moderate dimensional underlying process, deterministic (PDE) based methods may be applicable, for higher dimensions Monte Carlo simulation based methods are virtually the only way out. Besides the absence of curse of dimensionality, Monte Carlo methods are quite popular because of their genericity. In the nineties a number of regression methods for constructing "good" exercise policies, hence price lower bounds, were introduced and studied in the literature (see Carriere (1996), Longstaff and Schwartz (2001), and Tsitsiklis and Van Roy (1999)). Among various other developments, we mention the stochastic mesh

\footnotetext{
${ }^{1}$ This research was partially supported by the Deutsche Forschungsgemeinschaft through the SPP 1324 "Mathematical methods for extracting quantifiable information from complex systems" and through Research Center Matheon "Mathematics for Key Technologies", and by Laboratory for Structural Methods of Data Analysis in Predictive Modeling, MIPT, RF government grant, ag. 11.G34.31.0073.

${ }^{2}$ Duisburg-Essen University, Duisburg, Germany \{denis.belomestny,fabian.dickmann\}@uni-due.de

${ }^{3}$ Weierstraß Institute (WIAS), Berlin, Germany schoenma@wias-berlin.de
} 
method of Broadie and Glasserman (2004), the quantization method by Bally and Pages (2003), and the policy iteration method by Kolodko and Schoenmakers (2006). The latter method may be effectively combined with the Longstaff-Schwartz approach as presented in Bender et al. (2008).

The aforementioned methods have in common that they provide an exercise policy for the American product. Based on this policy one may simulate a lower bound for its price. This is what is called the primal approach. A new direction in Monte Carlo simulation of American options was the dual approach, developed by Rogers (2002) and independently by Haugh and Kogan (2004), related to earlier ideas in Davis and Karatzas (1994). In this approach one looks for a "good" martingale rather than a "good" stopping time. Based on this martingale the price of an American derivative may be bounded from above. In fact, this price upper bound may be represented by a "look-back" option due to the difference of the cash-flow and the martingale. Meanwhile, several numerical algorithms for computing dual upper bounds have appeared in the literature. Probably one of the most popular ones is the method of Andersen and Broadie (2004). A drawback of this method is its computational complexity due to the need for nested Monte Carlo simulation. In recent years a number of approaches towards reducing the complexity of the $\mathrm{AB}$ algorithm appeared in the literature. As one remedy to the complexity issue, Belomestny et al. (2009) proposed a dual simulation algorithm which does not require nested simulation and uses regression to approximate the integrand in a martingale representation. Joshi (2007) suggested to exclude exercise at sub-optimal points to improve the efficiency of the $\mathrm{AB}$ algorithm. In Broadie and Cao (2008) a variance reduction approach based on martingale control variates was introduced. Another non-nested regression based dual algorithm was proposed in Schoenmakers et al. (2012) in the context of a study of optimal dual martingales (approximated by "low variance" martingales). Furthermore, in Belomestny (2012) an efficient dual algorithm is constructed which is based on convex optimization and empirical variance penalization.

Each of the above mentioned approaches can improve the efficiency of the $\mathrm{AB}$ algorithm significantly in some situations. However, none of them is provably uniformly superior (at least asymptotically) to the nested $\mathrm{AB}$ algorithm in terms of numerical complexity. For example, although the complexity of Belomestny et al. (2009) method is a multiple of the number of Monte Carlo paths $N$, the corresponding multiplicative constant is proportional to the number of basis functions used to approximate a target martingale. In order to ensure convergence, this number should go to infinity together with $N$ at some rate which in turn depends on the problem at hand.

The main goal of this paper is to enhance the efficiency of dual algorithms by using a "multilevel" idea in the spirit of Giles (2008). In Giles (2008) a multilevel Monte Carlo estimator is presented, which is based on approximate solutions of a stochastic differential equation given a sequence of different time discretization steps. For instance, by this method the complexity of simulating a European option can be significantly reduced. In this paper we apply the multilevel idea to a sequence of martingales (rather than time discretization steps). Based on this sequence of martingales we will construct a new multilevel dual estimator for the American option. As a special case we obtain a multilevel version of the Andersen-Broadie algorithm. Under some assumptions we will prove that the complexity of this algorithm is (almost) equivalent to a non-nested Monte Carlo algorithm. As a byproduct of our complexity analysis we derive, to our knowledge for the first time, convergence rates of the Andersen-Broadie algorithm. In particular, our analysis reveals that, under some assumptions, the upper bias induced by inner simulations 
converges to zero at rate $O(1 / k)$, with $k$ being the number of inner simulations. The latter feature was observed empirically in the early literature (see, e.g., Kolodko and Schoenmakers (2004)), but has not yet had a theoretical explanation.

The structure of the paper is as follows. In Section 2 we recap the primal and dual approaches for optimal stopping in the context of American options. The main setup and prerequisites including a key Theorem 5 are presented in Section 3. After a complexity analysis of the standard dual approach in Section 4 we present and analyze the multi-level dual estimator in Section 5. Section 6 concludes with a detailed numerical study of the multilevel version of the Andersen-Broadie algorithm.

\section{Primal and Dual valuation of American options}

Let $\left(Z_{j}\right)_{j \geq 0}$ be a nonnegative adapted process on a filtered probability space $\left(\Omega, \mathbb{F}=\left(\mathcal{F}_{j}\right)_{j \geq 0}, \mathrm{P}\right)$ representing the discounted payoff of an American option, so that the holder of the option receives $Z_{j}$ if the option is exercised at time $j \in\{0, \ldots, \mathcal{J}\}$ with $\mathcal{J} \in \mathbb{N}_{+}$. The pricing of American options can be formulated as a primal-dual problem. Let $Y_{j}$ denote the time $j$ solution to this problem. The primal representation corresponds to the following optimal stopping problems:

$$
Y_{j}^{*}=\max _{\tau \in \mathcal{T}[j, \ldots, \mathcal{J}]} \mathrm{E}_{\mathcal{F}_{j}}\left[Z_{\tau}\right], \quad j=0, \ldots, \mathcal{J},
$$

where $\mathcal{T}[j, \ldots, \mathcal{J}]$ is the set of $\mathbb{F}$-stopping times taking values in $\{j, \ldots, \mathcal{J}\}$. During the nineties the primal approach was the only method available. More recently a quite different "dual" approach has been discovered by Rogers (2002) and Haugh and Kogan (2004). The next theorem summarizes their results.

Theorem 1 Let $\mathcal{M}$ denote the space of adapted martingales, then we have the following dual representation for the value process $Y_{j}^{*}$

$$
\begin{aligned}
Y_{j}^{*} & =\inf _{M \in \mathcal{M}} \mathrm{E}_{\mathcal{F}_{j}}\left[\max _{s \in\{j, \ldots, \mathcal{J}\}}\left(Z_{s}-M_{s}+M_{j}\right)\right] \\
& =\max _{s \in\{j, \ldots, \mathcal{J}\}}\left(Z_{s}-M_{s}^{*}+M_{j}^{*}\right) \quad \text { a.s. }
\end{aligned}
$$

where

$$
Y_{j}^{*}=Y_{0}^{*}+M_{j}^{*}-A_{j}^{*}
$$

is the (unique) Doob decomposition of the supermartingale $Y_{j}^{*}$. That is, $M^{*}$ is a martingale and $A^{*}$ is an increasing process with $M_{0}=A_{0}=0$ such that (1) holds.

Remark 2 In Schoenmakers et al. (2012) it is shown that in general there are infinitely many martingales $M^{\circ}$ such that

$$
Y_{j}^{*}=\max _{s \in\{j, \ldots, \mathcal{J}\}}\left(Z_{s}-M_{s}^{\circ}+M_{j}^{\circ}\right) \quad \text { a.s. }
$$


Theorem 1 implies that, for an arbitrarily chosen martingale $M$ with $M_{0}=0$, the value

$$
\mathrm{E}\left[\max _{s \in\{0, \ldots, \mathcal{J}\}}\left(Z_{s}-M_{s}\right)\right]
$$

defines an upper bound for the price of American option $Y_{0}^{*}$, and the upper bound will be tight if the chosen martingale $M$ is close to the Doob martingale part $M^{*}$ of the discounted true value process $Y_{j}^{*}$, which we shall refer to as the "optimal" martingale. Not surprisingly, finding such an optimal martingale is no easier than solving the original stopping problem. The so-called martingale duality approach aims at finding a martingale that approximates the "optimal" martingale and then use this approximation to compute an upper bound for the price of the American option by Monte Carlo. There are several methods known for approximating the "optimal" martingale $M^{*}$. In Andersen and Broadie (2004) the Doob martingale part of a given approximation to the Snell envelope is constructed by sub-simulations. In this way quite tight lower and upper bounds for a number of test examples were obtained. However, as we will see later on, the complexity of this method can be rather high, especially if a high precision of calculation is required. Therefore the complexity reduction of the dual algorithms is of primal importance and is one of the main aims of this paper.

\section{Main setup, prerequisites, and central results}

Let $\left(M^{k}\right)_{k \in \mathbb{N}}$ be a sequence of $\mathbb{F}^{\prime}$-martingales starting at $0\left(M_{0}^{k}=0\right)$ with respect to an enlarged probability space $\left(\Omega, \mathbb{F}^{\prime}=\left(\mathcal{F}_{j}^{\prime}\right)_{j \geq 0}, P\right)$, where $\mathcal{F}_{j} \subset \mathcal{F}_{j}^{\prime}$ for each $j$. It is supposed that the sequence $\left(M^{k}\right)_{k \in \mathbb{N}}$ converges in some sense to a target martingale $M$ which is $\mathbb{F}$-adapted. The need for an enlarged probability space in order to approximate the martingale $M$ is best illustrated in the following canonical situation.

Example 3 Let $Y=\left(Y_{j}\right)_{0 \leq j \leq \mathcal{J}}$ be some given $\mathbb{F}$-adapted process and $M=\left(M_{j}\right)_{0 \leq j \leq \mathcal{J}}$ be the Doob martingale corresponding to $Y$, i.e.,

$$
M_{j}=\sum_{i=1}^{j}\left(Y_{i}-\mathrm{E}_{\mathcal{F}_{i-1}}\left[Y_{i}\right]\right) .
$$

A typical way to obtain an approximation $M^{k}$ to $M$ is to estimate the conditional expectations in (2) by Monte Carlo simulation within the following setup. Consider

$$
M_{j}^{k}=\sum_{i=1}^{j}\left(Y_{i}-\frac{1}{k} \sum_{l=1}^{k} \xi_{i}^{(l)}\right),
$$

where, conditionally $\mathbb{F}$, all random variables $\xi_{i}^{(l)}, l=1, \ldots, k, i=1, \ldots, \mathcal{J}$, are independent and moreover, for each particular $i$, conditionally i.i.d. for $1 \leq l \leq k$, such that the $\mathbb{F}$-conditional law of $\xi_{i}^{(1)}$ equals its $\mathcal{F}_{i-1}$-conditional law, and such that

$$
\mathrm{E}_{\mathbb{F}}\left[\xi_{i}^{(l)}\right]=\mathrm{E}_{\mathcal{F}_{i-1}}\left[\xi_{i}^{(l)}\right]=\mathrm{E}_{\mathcal{F}_{i-1}}\left[\xi_{i}^{(1)}\right]=\mathrm{E}_{\mathcal{F}_{i-1}}\left[Y_{i}\right] .
$$

In this setup we obviously have that $M^{k}$ is an $\mathbb{F}^{\prime}$-martingale with $\mathcal{F}_{j}^{\prime}=\mathcal{F}_{j} \vee \sigma\left\{\xi_{p}^{(l)}, p=1, \ldots, j\right.$, $l=1, \ldots k\}$. 
Our aim is to approximate the target upper bound:

$$
Y(M):=\mathrm{E}\left[\max _{j=0, \ldots, \mathcal{J}}\left(Z_{j}-M_{j}\right)\right]=\mathrm{E}[\mathcal{Z}(M)]
$$

with

$$
\mathcal{Z}(M)=\max _{j=0, \ldots, \mathcal{J}}\left(Z_{j}-M_{j}\right) .
$$

Note that any $\mathbb{F}$-stopping time $\tau$ is also an $\mathbb{F}^{\prime}$-stopping time, so by Doob's sampling theorem $\mathrm{E}\left[M_{\tau}^{k}\right]=0$, and we have

$$
\begin{aligned}
Y_{0}^{*} & =\sup _{\mathbb{F} \text {-stopping times } \tau} \mathrm{E}\left[Z_{\tau}\right] \\
& =\sup _{\mathbb{F} \text {-stopping times } \tau} \mathrm{E}\left[Z_{\tau}-M_{\tau}^{k}\right] \\
& \leq \mathrm{E}\left[\max _{j=0, \ldots, \mathcal{J}}\left(Z_{j}-M_{j}^{k}\right)\right]=Y\left(M^{k}\right),
\end{aligned}
$$

i.e., $Y\left(M^{k}\right)$ is an upper bound for any $k \in \mathbb{N}$.

Remark 4 In the setting of Example 3 we have that $Y\left(M^{k}\right)$ is upper biased with respect to $Y(M)$. Indeed, by Jensen's inequality it follows that

$$
Y\left(M^{k}\right) \geq \mathrm{E}\left[\max _{j=0, \ldots, \mathcal{J}}\left(Z_{j}-\mathrm{E}_{\mathbb{F}}\left[M_{j}^{k}\right]\right)\right]=\mathrm{E}\left[\max _{j=0, \ldots, \mathcal{J}}\left(Z_{j}-M_{j}\right)\right]=Y(M) .
$$

We are now interested in the convergence behavior of $\mathcal{Z}\left(M^{k}\right)$ to $\mathcal{Z}(M)$ as $k \rightarrow \infty$, and consider in this context the following set of assumptions.

(AC) The numerical complexity of obtaining a single realization of $M_{j}^{k}$ is of order $O(k)$ for each $j=1, \ldots, \mathcal{J}$.

It should be noted that $(\mathrm{AC})$ is not really an assumption as it can always be achieved after a suitable re-parametrization of the martingale sequence. The next two assumptions describe the rate of convergence to the target martingale.

(AR) There exists an $\mathbb{F}$-adapted martingale $M$ such that

$$
\mathrm{E}\left[\max _{j=0, \ldots, \mathcal{J}}\left(M_{j}^{k}-M_{j}\right)^{2}\right] \leq B k^{-\beta}, \quad k \in \mathbb{N},
$$

for some $\beta>0$ and $B>0$.

(AR') There exists an $\mathbb{F}$-adapted martingale $M$ such that

$$
\max _{j=0, \ldots, \mathcal{J}}\left|\mathrm{E}_{\mathbb{F}}\left[M_{j}^{k}-M_{j}\right]\right| \leq A k^{-\alpha}, \quad \mathrm{E}_{\mathbb{F}}\left[\max _{j=0, \ldots, \mathcal{J}}\left(M_{j}^{k}-M_{j}\right)^{2}\right] \leq B k^{-\beta},
$$

$\mathbb{F}$-almost surely, for all $k \in \mathbb{N}$, some $\beta>0, \alpha \geq \beta / 2, A>0$ and $B>0$. 
Let us further consider the random sets

$$
\mathcal{Q}=\left\{j: Z_{j}-M_{j}=\mathcal{Z}(M)\right\}, \quad \mathcal{Q}^{k}=\left\{j: Z_{j}-M_{j}^{k}=\mathcal{Z}\left(M^{k}\right)\right\}, \quad k \in \mathbb{N},
$$

and define the $\mathbb{F}$-measurable random variable

$$
\Lambda:=\min _{j \notin \mathcal{Q}}\left(\mathcal{Z}(M)-Z_{j}+M_{j}\right),
$$

with $\Lambda:=+\infty$ if $\mathcal{Q}=\{0, \ldots, \mathcal{J}\}$. We then introduce the following two conditions.

(AL) $\Lambda$ satisfies $\mathrm{E}\left[\Lambda^{-a}\right]<\infty$, for some $a>0$.

(AQ) $\# \mathcal{Q}=1$ a.s.

In terms of the different assumptions spelled out above, we now have the following central theorem which is proved in Section 7.1.

Theorem 5 Under assumption (AR) alone it holds that

$$
\left|\mathrm{E}\left[\mathcal{Z}\left(M^{k}\right)-\mathcal{Z}(M)\right]\right| \leq C k^{-\gamma}, \quad \mathrm{E}\left[\left(\mathcal{Z}\left(M^{k}\right)-\mathcal{Z}(M)\right)^{2}\right] \leq B k^{-\beta}
$$

with $\gamma=\beta / 2$ and some $C>0$. If assumptions (AR'), (AL) and (AQ) are additionally satisfied, then (5) holds true with $\gamma=\min \{\alpha, \beta \min \{1,(a+1) / 2\}\}$.

Discussion (Andersen \& Broadie algorithm) Example 3 basically describes the setup of the Andersen and Broadie (2004) (AB) algorithm. In this algorithm, $M$ is the Doob martingale part of some approximation $Y$ to the Snell envelope, and the sequence of martingales $\left(M^{k}\right)$ is constructed using sub-simulations, where for each $i$ the random variables $\xi_{i}^{(l)}$ are simulated as i.i.d. copies according to the distribution of $Y_{i}$, conditionally on $\mathcal{F}_{i-1}$. It is not difficult to see that for the $\mathrm{AB}$ algorithm (AR) holds with $\beta=1$, provided $\mathrm{E}\left[Y_{i}^{2}\right]<\infty$ for $i=0, \ldots, \mathcal{J}$. Indeed, since all $\xi_{i}^{(l)}$ are independent conditionally $\mathbb{F}$, it holds for each $j$,

$$
\begin{aligned}
\mathrm{E}\left[\left(M_{j}^{k}-M_{j}\right)^{2}\right] & =\mathrm{E}_{\mathbb{F}}\left[\left\{\sum_{i=1}^{j}\left(\mathrm{E}_{\mathcal{F}_{i-1}}\left[Y_{i}\right]-\frac{1}{k} \sum_{l=1}^{k} \xi_{i}^{(l)}\right)\right\}^{2}\right] \\
& =\frac{1}{k} \sum_{i=1}^{\mathcal{J}} \mathrm{E} \mathrm{E}_{\mathcal{F}_{i-1}}\left[\left(\mathrm{E}_{\mathcal{F}_{i-1}}\left[Y_{i}\right]-\xi_{i}^{(1)}\right)^{2}\right] \leq \frac{1}{k} \sum_{i=1}^{\mathcal{J}} \mathrm{E}\left[Y_{i}^{2}\right] .
\end{aligned}
$$

In the $\mathrm{AB}$ algorithm the estimator $M^{k}$ (cf. (3)) is bias-free, so the first part of (AR') holds with $\alpha=\infty$. Further if, for example, the proxy process $Y$ in the AB algorithm is almost surely bounded, a similar argumentation as above shows that (AR') holds with $\beta=1$. A bounded proxy process is for instance easily obtained when the cash-flow process $Z_{j}, j=0, \ldots, \mathcal{J}$, is bounded. 


\section{Standard Monte Carlo dual upper bound}

Fix some natural numbers $N$ and $K$, and consider the estimator:

$$
\begin{aligned}
Y^{N, K} & =\frac{1}{N} \sum_{n=1}^{N} \max _{j=0, \ldots, \mathcal{J}}\left(Z_{j}^{(n)}-M_{j}^{K,(n)}\right) \\
& =: \frac{1}{N} \sum_{n=1}^{N} \mathcal{Z}^{(n)}\left(M^{K}\right)
\end{aligned}
$$

based on a set of trajectories

$$
\left\{\left(Z_{j}^{(n)}, M_{j}^{K,(n)}\right), n=1, \ldots, N, j=0, \ldots, \mathcal{J}\right\}
$$

of the vector process $\left(Z, M^{K}\right)$. Let us study the numerical complexity of $Y^{N, K}$.

\subsection{Complexity analysis}

Under assumption (AR) only we have due to Theorem 5 that for some $C>0$

$$
\begin{aligned}
\mathrm{E}\left[\left(Y^{N, K}-Y(M)\right)^{2}\right] & \leq N^{-1} \operatorname{Var}\left[\mathcal{Z}\left(M^{K}\right)\right]+C K^{-\beta} \\
& =: N^{-1} v_{K}+C K^{-\beta}, \quad K \rightarrow \infty .
\end{aligned}
$$

In order to obtain $\sqrt{\mathrm{E}\left[\left(Y^{N, K}-Y(M)\right)^{2}\right]} \leq \varepsilon$, we thus may take

$$
K=\left\lceil\frac{(2 C)^{1 / \beta}}{\varepsilon^{2 / \beta}}\right\rceil, \quad \text { and next } \quad N=\left\lceil\frac{2 v_{K}}{\varepsilon^{2}}\right\rceil
$$

with $\lceil x\rceil$ denoting the first integer which is larger than or equal to $x$. Assuming that $v_{K}$ is non-increasing, the required computation time to reach the accuracy $\varepsilon$, hence the complexity, is then given, up to a constant, by

$$
\mathcal{C}^{N, K}(\varepsilon):=N K=O\left(\frac{\left.v \frac{(2 C)^{1 / \beta}}{\varepsilon^{2 / \beta}}\right\rceil}{\varepsilon^{2+2 / \beta}}\right) .
$$

In the usual case, where $M$ is the Doob martingale of some approximation $Y$ to the Snell envelope $Y^{*}$, we have that $\operatorname{Var}[\mathcal{Z}(M)]>0$ and $v_{K} \rightarrow v_{\infty} \neq 0$ leading to $\mathcal{C}^{N, K}(\varepsilon)$ of order $O\left(\varepsilon^{-2-2 / \beta}\right)$, hence $O\left(\varepsilon^{-4}\right)$ for the AB algorithm. As the following example shows, the AB algorithm may have rate $\varepsilon^{-4}$ in general indeed.

Example 6 Consider the simple situation where $\mathcal{J}=2, Z_{0}=Z_{1}=0, Y_{0}^{*}=0$ and $M_{0}^{*}=M_{1}^{*}=$ 0 . Define a target martingale via $M_{0}=0, M_{1}=Y_{1}-\mathrm{E} Y_{1}=\xi-\mathrm{E}[\xi]$ with a r.v. $\xi$ given by

$$
\xi= \begin{cases}3 b / 2 & \text { with probability } 1 / 4 \\ b & \text { with probability } 1 / 2 \\ b / 2 & \text { with probability } 1 / 4\end{cases}
$$


for some $b>0$. Note that $E[\xi]=b$ and $\operatorname{Var}[\xi]=b^{2} / 8$. The approximation

$$
M_{1}^{K}=\xi-\frac{1}{K} \sum_{l=1}^{K} \xi^{(l)}
$$

where $\xi^{(l)}$ are i.i.d. copies of $\xi$, gives us

$$
\mathcal{Z}\left(M^{K}\right)=\max \left(0,-M_{1}^{K}\right)=\max \left(0, \frac{1}{K} \sum_{l=1}^{K} \xi^{(l)}-\xi\right)
$$

Hence

$$
\Delta_{K}:=\mathrm{E}\left[\mathcal{Z}\left(M^{K}\right)\right]-\mathrm{E}[\mathcal{Z}(M)]=\mathrm{E}\left[\left(\frac{1}{K} \sum_{l=1}^{K} \xi^{(l)}-\xi\right)^{+}-(\mathrm{E} \xi-\xi)^{+}\right]
$$

and it is easy to check that

$$
\Delta_{K} \geq \frac{1}{2} \mathrm{E}\left[\left(\frac{1}{K} \sum_{l=1}^{K} \xi^{(l)}-b\right)^{+}\right]=\frac{b / \sqrt{8}}{2 \sqrt{K}} \mathrm{E}\left[\left(\frac{1}{\sqrt{K}} \sum_{l=1}^{K} \frac{\xi^{(l)}-b}{b / \sqrt{8}}\right)^{+}\right] \asymp \frac{b}{4 \sqrt{\pi K}}
$$

as $K \rightarrow \infty$. Moreover, it obviously holds

$$
\mathrm{E}\left[\left(\mathcal{Z}\left(M^{K}\right)-\mathcal{Z}(M)\right)^{2}\right]=O(1 / K), \quad K \rightarrow \infty .
$$

Thus, (AR) holds with $\beta=1$ and the decay of the bias is proportional to $K^{-\beta / 2}$ leading to the complexity rate $\varepsilon^{-4}$ for the $A B$ algorithm in this case.

Remark 7 It is easy to see that in Example $6(A L)$ is violated for all $a>0$.

Remark 8 If $\operatorname{Var}[\mathcal{Z}(M)]=0$ (e.g., the target martingale $M$ is the Doob martingale of $Y^{*}$ ) we have

$$
v_{K}=\operatorname{Var}\left[\mathcal{Z}\left(M^{K}\right)\right] \leq \mathrm{E}\left[\left(\mathcal{Z}\left(M^{K}\right)-\mathcal{Z}(M)\right)^{2}\right] \leq B K^{-\beta},
$$

and as a result

$$
\mathcal{C}^{N, K}(\varepsilon)=O\left(\frac{1}{\varepsilon^{2 / \beta}}\right)
$$

That is, if $\beta \geq 1$ the complexity of the $A B$ algorithm is even less than or equal to the complexity of the plain Monte Carlo algorithm!

Under the more restrictive assumptions (AR'), (AL), and (AQ), Theorem 5 yields (cf. (6))

$$
\mathrm{E}\left[\left(Y^{N, K}-Y(M)\right)^{2}\right] \leq N^{-1} v_{K}+C K^{-2 \gamma}, \quad K \rightarrow \infty,
$$

so in order to get $\sqrt{\mathrm{E}\left[\left(Y^{N, K}-Y(M)\right)^{2}\right]} \leq \varepsilon$, we then may take

$$
K=\left\lceil\frac{(2 C)^{1 / 2 \gamma}}{\varepsilon^{1 / \gamma}}\right\rceil, \quad N=\left\lceil\frac{2 v_{K}}{\varepsilon^{2}}\right\rceil, \quad \text { yielding } \quad \mathcal{C}^{N, K}(\varepsilon)=O\left(\frac{v\left[\frac{(2 C)^{1 / 2 \gamma}}{\varepsilon^{1 / \gamma}}\right\rceil}{\varepsilon^{2+1 / \gamma}}\right)
$$


Corollary 9 Under ( $\left.A R^{\prime}\right),(A L)$, and $(A Q)$ the complexity of the standard AB algorithm, where $\alpha=\infty, \beta=1$, may be reduced to

$$
O\left(\frac{1}{\varepsilon^{2+\frac{2}{a+1}}}\right)
$$

in the case $v_{K} \rightarrow v_{\infty} \neq 0$, and $0<a \leq 1$. That is, the $A B$ complexity is of order $O\left(\varepsilon^{-3-\delta}\right)$ for arbitrary small $\delta>0$.

\section{Discussion}

- (AL) is fulfilled for all $0 \leq a<q+1$ when $\Lambda>0$ has density $g$ in a neighborhood of zero such that $g(z)=O\left(|z|^{q}\right)$ for some $q \geq 0$ as $|z| \rightarrow 0$.

- If $M=M^{*}$ then, using (1), (4), and the fact that $Y_{0}^{*}=\mathcal{Z}\left(M^{*}\right)$,

$$
\begin{aligned}
\Lambda & =\min _{j \notin \mathcal{Q}}\left(Y_{0}^{*}-Z_{j}+M_{j}^{*}\right) \\
& =\min _{j \notin \mathcal{Q}}\left(Y_{j}^{*}-Z_{j}+A_{j}^{*}\right) \\
& \geq \min _{j \notin \mathcal{Q}}\left(Y_{j}^{*}-Z_{j}\right) \geq \min _{\left\{j: Y_{j}^{*}>Z_{j}\right\}}\left(Y_{j}^{*}-Z_{j}\right) .
\end{aligned}
$$

Hence, the condition (AL) is fulfilled if

$$
\mathrm{P}\left(Y_{j}^{*}-Z_{j}<\delta \mid Y_{j}^{*}>Z_{j}\right) \lesssim \delta^{q}, \quad \delta \rightarrow 0,
$$

for all $j=1, \ldots, \mathcal{J}$ and some $q>a$. The condition (7) bounds the probability of staying in the $\delta$-vicinity of the exercise boundary $\left\{Y_{j}^{*} \leq Z_{j}\right\}$ in the case of continuation and is similar to the so-called margin condition in Belomestny (2011). As we will see, (7) leads to faster convergence rates of the standard Andersen-Broadie dual algorithm.

Remark 10 Under the additional assumptions ( $A L)$ with $a \leq 1$, and (AQ), we have by Theorem 5 that the bias of the Andersen-Broadie estimator is of order $O\left(k^{-(1+a) / 2}\right)$. In Kolodko and Schoenmakers (2004) a rate of order $O\left(k^{-1}\right)$ was experimentally detected in experiments but before now, to the best of our knowledge, no theoretical justification for this observation was given.

In the next section we propose a multi-level Monte-Carlo algorithm for computing $Y(M)$ whose complexity is under (AR) with $\beta=1$ of order $O\left(\varepsilon^{-2} \ln ^{2}(\varepsilon)\right)$, regardless whether (AR'), $(A L)$, and $(A Q)$ do hold or not!

\section{Multilevel dual algorithm}

Fix some natural number $L>0$. Let $\mathbf{k}=\left(k_{0}, \ldots, k_{L}\right)$ be a sequence of natural numbers satisfying $1 \leq k_{0}<k_{1}<\ldots<k_{L}$. Write

$$
\begin{aligned}
Y\left(M^{k_{L}}\right) & =Y\left(M^{k_{0}}\right)+\sum_{l=1}^{L}\left[Y\left(M^{k_{l}}\right)-Y\left(M^{k_{l-1}}\right)\right] \\
& =\mathrm{E}\left[\mathcal{Z}\left(M^{k_{0}}\right)\right]+\sum_{l=1}^{L} \mathrm{E}\left[\mathcal{Z}\left(M^{k_{l}}\right)-\mathcal{Z}\left(M^{k_{l-1}}\right)\right] .
\end{aligned}
$$


For a given sequence $\mathbf{n}=\left(n_{0}, \ldots, n_{L}\right)$ with $n_{0}>\ldots>n_{L} \geq 1$, we first simulate the initial set of trajectories

$$
\left\{\left(Z_{j}^{(i)}, M_{j}^{k_{0},(i)}\right), \quad i=1, \ldots, n_{0}, \quad j=0, \ldots, \mathcal{J}\right\}
$$

of the vector process $\left(Z, M^{k_{0}}\right)$, and then for each level $l=1, \ldots, L$ independently a set of trajectories

$$
\left\{\left(Z_{j}^{(i)}, M_{j}^{k_{l-1},(i)}, M_{j}^{k_{l},(i)}\right), \quad i=1, \ldots, n_{l}, \quad j=0, \ldots, \mathcal{J}\right\}
$$

of the vector process $\left(Z, M^{k_{l-1}}, M^{k_{l}}\right)$. Based on these simulations we consider the following approximation of (8)

$$
Y^{\mathbf{n}, \mathbf{k}}:=\frac{1}{n_{0}} \sum_{i=1}^{n_{0}} \mathcal{Z}^{(i)}\left(M^{k_{0}}\right)+\sum_{l=1}^{L} \frac{1}{n_{l}} \sum_{i=1}^{n_{l}}\left[\mathcal{Z}^{(i)}\left(M^{k_{l}}\right)-\mathcal{Z}^{(i)}\left(M^{k_{l-1}}\right)\right]
$$

with $\mathcal{Z}^{(i)}\left(M^{k}\right):=\max _{j=0, \ldots, \mathcal{J}}\left(Z_{j}^{(i)}-M_{j}^{k,(i)}\right), i=1, \ldots, n_{l}, k \in \mathbb{N}$, where $M^{k,(i)}$ denotes the $i$-th simulated trajectory of the martingale $M^{k}$.

\subsection{Complexity analysis}

For the bias of the multilevel estimator we obtain by Theorem 5

$$
\left|\mathrm{E}\left[Y^{\mathbf{n}, \mathbf{k}}\right]-Y(M)\right|=\left|\mathrm{E}\left[\mathcal{Z}\left(M^{k_{L}}\right)\right]-\mathrm{E}[\mathcal{Z}(M)]\right| \leq C k_{L}^{-\gamma}
$$

and for the variance we have

$$
\operatorname{Var}\left[Y^{\mathbf{n}, \mathbf{k}}\right]=n_{0}^{-1} \operatorname{Var}\left[\mathcal{Z}\left(M^{k_{0}}\right)\right]+\sum_{l=1}^{L} \frac{1}{n_{l}} \operatorname{Var}\left[\mathcal{Z}\left(M^{k_{l}}\right)-\mathcal{Z}\left(M^{k_{l-1}}\right)\right] .
$$

Note that for any $l>0$,

$$
\begin{aligned}
\operatorname{Var}\left[\mathcal{Z}\left(M^{k_{l}}\right)-\mathcal{Z}\left(M^{k_{l-1}}\right)\right] & \leq \mathrm{E}\left[\left(\mathcal{Z}\left(M^{k_{l}}\right)-\mathcal{Z}\left(M^{k_{l-1}}\right)\right)^{2}\right] \\
& \leq 2 \mathrm{E}\left[\left(\mathcal{Z}\left(M^{k_{l}}\right)-\mathcal{Z}(M)\right)^{2}\right] \\
& +2 \mathrm{E}\left[\left(\mathcal{Z}\left(M^{k_{l-1}}\right)-\mathcal{Z}(M)\right)^{2}\right] \\
& \leq 2\left(B k_{l}^{-\beta}+B k_{l-1}^{-\beta}\right) \leq 4 B k_{l}^{-\beta} \leq \widetilde{B} k_{l}^{-\beta},
\end{aligned}
$$

by Theorem 5 . For notational convenience we assume that $\widetilde{B}$ is such that $\operatorname{Var}\left[\mathcal{Z}\left(M^{k_{0}}\right)\right] \leq \widetilde{B} k_{0}^{-\beta}$.

Theorem 11 (complexity theorem) Suppose that $k_{l}=k_{0} \kappa^{l}$ for some integer $k_{0}, \kappa>1$, and $l=0, \ldots, L$. Assume that the inequalities (5) hold with $\gamma \geq 1 / 2$. Fix some $0<\varepsilon<1$ and set

$$
L=\left\lceil\frac{-\ln \frac{k_{0}^{\gamma} \varepsilon}{C \sqrt{2}}}{\gamma \ln \kappa}\right\rceil .
$$


Let

$$
n_{l}= \begin{cases}{\left[2 \varepsilon^{-2} \widetilde{B} k_{0}^{-\beta} \kappa^{L(1-\beta) / 2}\left(1-\kappa^{-(1-\beta) / 2}\right)^{-1} \kappa^{-l(1+\beta) / 2}\right\rceil,} & \beta<1, \\ \left.2 \varepsilon^{-2} \widetilde{B}(L+1) k_{0}^{-1} \kappa^{-l}\right\rceil, & \beta=1, \\ \left.\mid 2 \varepsilon^{-2} \widetilde{B} k_{0}^{-\beta}\left(1-\kappa^{-(\beta-1) / 2}\right)^{-1} \kappa^{-l(1+\beta) / 2}\right\rceil, & \beta>1 .\end{cases}
$$

Then

$$
\sqrt{\mathrm{E}\left[\left(Y^{\mathbf{n}, \mathbf{k}}-Y(M)\right)^{2}\right]} \leq \varepsilon,
$$

while the computational complexity of the estimator $Y^{\mathbf{n}, \mathbf{k}}$ is, up to a constant, given by

$$
\mathcal{C}^{\mathbf{n}, \mathbf{k}}(\varepsilon):=\sum_{l=0}^{L} k_{l} n_{l}= \begin{cases}O\left(\varepsilon^{-2-(1-\beta) / \gamma}\right), & \beta<1 \\ O\left(\varepsilon^{-2} \ln ^{2} \varepsilon\right), & \beta=1 \\ O\left(\varepsilon^{-2}\right), & \beta>1\end{cases}
$$

\subsection{Refined complexity analysis}

While the general complexity analysis in Section 5.1 provides the multilevel complexity order for achieving a specific accuracy $\varepsilon$, it will be advantageous to refine this analysis in the present context in order to optimize order coefficients in this respect. The idea is a separate treatment of $n_{0}$ in the first term of (8). Let us therefore assume that

$$
k_{l}=k_{0} \kappa^{l} \quad \text { for } \quad 0 \leq l \leq L, \quad \text { and } \quad n_{l}=n_{1} \kappa^{1-l} \quad \text { for } \quad 1 \leq l \leq L,
$$

for some separate integers $n_{1}$ and $n_{0}$ to be determined. Further it is assumed that the $k_{l-1}$ sub-simulations used for computing $\mathcal{Z}\left(M^{k_{l-1}}\right)$ are reused in the computation of $\mathcal{Z}\left(M^{k_{l}}\right)$. Then the numerical complexity of the multi-level estimate $Y^{\mathbf{n}, \mathbf{k}}$ is given, up to a constant, by

$$
\begin{aligned}
\mathcal{C}_{M L} & =n_{0} k_{0}+\sum_{l=1}^{L} n_{l} k_{l} \\
& =n_{0} k_{0}+n_{1} k_{0} \kappa L .
\end{aligned}
$$

In the analysis below we assume that the bias of the Andersen-Broadie algorithm is given by

$$
\mathbb{E}\left[Y^{\mathbf{n}, \mathbf{k}}-\widehat{Y}\right]=\frac{\mu_{\infty}}{k_{0} \kappa^{L}} .
$$

Let us now analyze the variance of $Y^{\mathbf{n}, \mathbf{k}}$. For this we assume that there are constants $\sigma_{\infty}^{2}$ and $\mathfrak{V}_{\infty}$ such that

$$
\begin{aligned}
\operatorname{Var}\left[Y^{\mathbf{n}, \mathbf{k}}\right] & =n_{0}^{-1} \operatorname{Var}\left[\mathcal{Z}\left(M^{k_{0}}\right)\right]+\sum_{l=1}^{L} n_{l}^{-1} \operatorname{Var}\left[\mathcal{Z}\left(M^{k_{l}}\right)-\mathcal{Z}\left(M^{k_{l-1}}\right)\right] \\
& =n_{0}^{-1} \sigma_{\infty}^{2}+\sum_{l=1}^{L} n_{l}^{-1} k_{l}^{-1} \mathfrak{V}_{\infty} \\
& =n_{0}^{-1} \sigma_{\infty}^{2}+n_{1}^{-1} k_{0}^{-1} \mathfrak{V}_{\infty} \kappa^{-1} L .
\end{aligned}
$$


For fixed $L, k_{0}$ and accuracy $\varepsilon$, we are now going to minimize the complexity (14) over $n_{0}$ and $n_{1}$ under the constraints

$$
\underbrace{\left(\frac{\mu_{\infty}}{k_{0} \kappa^{L}}\right)^{2}}_{\text {squared bias }}+\underbrace{n_{0}^{-1} \sigma_{\infty}^{2}+n_{1}^{-1} k_{0}^{-1} \mathfrak{V}_{\infty} \kappa^{-1} L}_{\text {variance }}=\varepsilon^{2},
$$

for fixed $L$ and $k_{0}$ such that the bias $\mu_{\infty} k_{0}^{-1} \kappa^{-L}<\varepsilon$, i.e.,

$$
L>\frac{\ln \varepsilon^{-1}+\ln \left(\mu_{\infty} / k_{0}\right)}{\ln \kappa} .
$$

A straightforward Lagrangian analysis (while treating integers as reals) leads to the following solution:

$$
\begin{aligned}
n_{0}^{*} & =n_{0}^{*}\left(k_{0}, L, \varepsilon\right)=\frac{\sigma_{\infty}^{2}+\sigma_{\infty} L \sqrt{k_{0}^{-1} \mathfrak{V}_{\infty}}}{\varepsilon^{2}-\mu_{\infty}^{2} k_{0}^{-2} \kappa^{-2 L}}, \\
n_{1}^{*} & =n_{1}^{*}\left(k_{0}, L, \varepsilon\right)=\frac{\sigma_{\infty} \kappa^{-1} \sqrt{k_{0}^{-1} \mathfrak{V}_{\infty}}+\kappa^{-1} L k_{0}^{-1} \mathfrak{V}_{\infty}}{\varepsilon^{2}-\mu_{\infty}^{2} k_{0}^{-2} \kappa^{-2 L}} \\
& =n_{0}^{*}\left(k_{0}, L, \varepsilon\right) \kappa^{-1} \sigma_{\infty}^{-1} \sqrt{k_{0}^{-1} \mathfrak{V}_{\infty}},
\end{aligned}
$$

corresponding to a complexity equal to

$$
\begin{aligned}
\mathcal{C}_{M L}^{*}\left(k_{0}, L, \varepsilon\right) & =k_{0} n_{0}^{*}\left(k_{0}, L, \varepsilon\right)+k_{0} \kappa L n_{1}^{*}\left(k_{0}, L, \varepsilon\right) \\
& =\frac{\left(L \sqrt{\mathfrak{V}_{\infty}}+\sigma_{\infty} \sqrt{k_{0}}\right)^{2}}{\varepsilon^{2}-\mu_{\infty}^{2} k_{0}^{-2} \kappa^{-2 L}} .
\end{aligned}
$$

Next, while keeping $k_{0}$ and $\varepsilon$ fixed, i.e. $\mathcal{C}_{M L}^{*}(L):=\mathcal{C}_{M L}^{*}\left(k_{0}, L, \varepsilon\right)$, we minimize (16) with respect to $L$ under the constraint (15). By considering the equation $d \mathcal{C}_{M L}^{*}(L) / d L=0$, we obtain after a little algebra, using $L>0$,

$$
\begin{aligned}
\kappa^{2 L} \varepsilon^{2} & =\sigma_{\infty} \mu_{\infty}^{2} \mathfrak{V}_{\infty}^{-1 / 2} k_{0}^{-3 / 2} \ln \kappa+\mu_{\infty}^{2} k_{0}^{-2}+L \mu_{\infty}^{2} k_{0}^{-2} \ln \kappa \\
& =: p+q L, \quad p, q>0 .
\end{aligned}
$$

So from (17) we see that necessarily $L \rightarrow \infty$ as $\varepsilon \downarrow 0$. Let us now reformulate (17) by taking logarithms as

$$
\begin{aligned}
L & =\frac{\ln \varepsilon^{-1}}{\ln \kappa}+\frac{1}{2 \ln \kappa} \ln (p+q L) \\
& =\frac{\ln \varepsilon^{-1}}{\ln \kappa}+\frac{\ln (q L)}{2 \ln \kappa}+O\left(L^{-1}\right), \quad \varepsilon \downarrow 0,
\end{aligned}
$$

which in turn may be written as

$$
L=\frac{\frac{\ln \varepsilon^{-1}}{\ln \kappa}+O\left(L^{-1}\right)}{1-\frac{\ln (q L)}{2 L \ln \kappa}},
$$


hence we have $L=O\left(\ln \varepsilon^{-1}\right)$, as $\varepsilon \downarrow 0$. This implies by (18),

$$
L=\frac{\ln \varepsilon^{-1}}{\ln \kappa}+O\left(\ln \ln \varepsilon^{-1}\right),
$$

and by iterating (18) once again using $q=\mu_{\infty}^{2} k_{0}^{-2} \ln \kappa$, we obtain

$$
L^{*}\left(k_{0}, \varepsilon\right):=\frac{\ln \varepsilon^{-1}}{\ln \kappa}+\frac{\ln \ln \varepsilon^{-1}}{2 \ln \kappa}+\frac{\ln \left(\mu_{\infty} / k_{0}\right)}{\ln \kappa}+O\left(\frac{\ln \ln \varepsilon^{-1}}{\ln \varepsilon^{-1}}\right), \quad \varepsilon \downarrow 0 .
$$

In fact, by iterating further in this way, we may obtain asymptotic expressions for the solution of (18) of arbitrary high order. Expression (19) is sufficient for our purposes however. Note that in (19) the order term goes to zero and the second term goes to infinity as $\varepsilon \downarrow 0$. Therefore, restriction (15) will be fulfilled provided $\varepsilon$ is not too large (the second term is positive for $\varepsilon<e^{-1}$ ), and then $\mathcal{C}_{M L}^{*}(L)$ will attain a minimum at the solution of (18). By plugging (19) into (16) we get for the minimized complexity after some standard asymptotic calculations

$$
\begin{gathered}
\mathcal{C}_{M L}^{*}\left(k_{0}, L, \varepsilon\right)= \\
\mathfrak{V}_{\infty} \varepsilon^{-2}\left(\frac{\ln \varepsilon^{-1}}{\ln \kappa}+\frac{\ln \ln \varepsilon^{-1}}{2 \ln \kappa}+\frac{\ln \left(\mu_{\infty} / k_{0}\right)}{\ln \kappa}+\frac{\sigma_{\infty} \sqrt{k_{0}}}{\sqrt{\mathfrak{V}_{\infty}}}\right)^{2}\left(1+O\left(\frac{\ln \ln \varepsilon^{-1}}{\ln \varepsilon^{-1}}\right)\right) .
\end{gathered}
$$

Discussion As the last pragmatic optimization step it looks natural to choose

$$
k_{0}^{*}=\frac{4 \mathfrak{V}_{\infty}}{\sigma_{\infty}^{2} \ln ^{2} \kappa}
$$

that minimizes the term involving $k_{0}$ in (20). However, this expression for $k_{0}^{*}$ should only be considered as an indicator, while it is better to choose $k_{0}$ by experience, for example $k_{0}=100$ turns out to be a robust choice. Further we observe that, asymptotically, the optimal value of $L$ depends on $\varepsilon$ and $\kappa$ only. Thus, as a recipe for a given $\varepsilon$, we propose to choose a suitable $k_{0}^{*}$, (e.g. 100 ), and then compute $L^{*}$ from (19), and next determine $n_{0}^{*}$ and $n_{1}^{*}$. For the Andersen-Broadie algorithm with $\beta=1$ we have

$$
\mathcal{C}_{A B}(\varepsilon)=\frac{2 \sqrt{2}}{\varepsilon^{-3}} \mu_{\infty} \sigma_{\infty}^{2},
$$

so the gain of the multilevel approach over the Andersen-Broadie algorithm, expressed as the ratio of their respective complexities, becomes

$$
\mathcal{R}(\varepsilon):=\frac{\mathcal{C}_{A B}(\varepsilon)}{\mathcal{C}_{M L}(\varepsilon)}=\frac{2 \sqrt{2} \ln ^{2} \kappa}{\varepsilon \ln ^{2} \varepsilon^{-1}} \theta
$$

with $\theta:=\mu_{\infty} \sigma_{\infty}^{2} / \mathfrak{V}_{\infty}$.

\section{Direct numerical comparison with $A B$}

In the next study we will compare the accuracies of the $\mathrm{ML}$ and $\mathrm{AB}$ algorithms given a certain computational budget, rather than comparing their complexities asymptotically based on the optimal choice of $L$ and the optimal allocations of $n_{l}$ and $k_{l}, l=0, \ldots, L$, for achieving a target accuracy $\varepsilon$ that tends to zero. Strictly speaking, we now have to seek for the optimal $L$ and the 
optimal allocations $n_{l}, k_{l}, l=0, \ldots, L$, given a cost budget $C$ for the ML algorithm, and next compare the achieved ML accuracy with the accuracy achieved by the AB algorithm based on the optimal allocation of $N$ and $K$, given the same cost budget $C$. In view of the complexity analysis given in Sections 5.1,5.2, it is not difficult to see that, for example, in the case $\beta=1$ and $\mathrm{AB}$ complexity of order $O\left(\varepsilon^{-3}\right)$, the ML accuracy $\varepsilon_{M L}$ is superior to the AB accuracy $\varepsilon_{A B}$ at a rate $\varepsilon_{M L} / \varepsilon_{A B}=O\left(C^{-1 / 6} \ln C\right)$ as $C \rightarrow \infty$. However, a further in depth analysis of the respective optimal parameters for the ML algorithm given a fixed budget $C$, is considered beyond the scope of this article. In order to show that the ML estimator is potentially advantageous compared to the $\mathrm{AB}$ estimator for a fixed (but finite) cost budget $C$, we propose the following simplified procedure. Let $N$ and $K$ be the (optimal) allocation for the AB method given some budget $C=N K$. We then choose in the multilevel approach $k_{0}$ and $L$ in such a way that $k_{L}=K$, i.e., we ensure that both methods have the same bias, and next show that by taking $L$ optimally the variance of the ML estimator may be smaller than the $\mathrm{AB}$ variance provided that $K$ (hence $C$ ) is large enough. The details are spelled out below.

\subsection{Parameter choice for the ML algorithm}

Let us recall the setting of Section 5.2 where the ML computational cost is given by (14) while the computational cost of the Andersen-Broadie estimate:

$$
Y^{K, N}=\frac{1}{N} \sum_{l=1}^{N} \mathcal{Z}^{(l)}\left(M^{K}\right)
$$

is equal to $N K$. Now we are going to compare the variances of the estimates $Y^{\mathbf{k}, \mathbf{n}}$ and $Y^{K, N}$, given that $Y^{\mathbf{k}, \mathbf{n}}$ and $Y^{K, N}$ have the same bias and the same computational cost. This leads to the following constraints:

$$
\begin{aligned}
N K & =n_{0} k_{0}+n_{1} k_{0} \kappa L \quad \text { equal cost } \\
k_{L} & =k_{0} \kappa^{L}=K \quad \text { equal bias }
\end{aligned}
$$

implying

$$
k_{0}=K \kappa^{-L}, \quad n_{1}=N L^{-1} \kappa^{L-1}-n_{0} \kappa^{-1} L^{-1} .
$$

The variance of $Y^{\mathbf{k}, \mathbf{n}}$ is given by

$$
\operatorname{Var}\left[Y^{\mathbf{k}, \mathbf{n}}\right]=\frac{v^{2}\left(0, k_{0}\right)}{n_{0}}+\sum_{l=1}^{L} n_{l}^{-1} v^{2}\left(k_{l-1}, k_{l}\right)
$$

with $v^{2}\left(k_{l-1}, k_{l}\right):=\operatorname{Var}\left[\mathcal{Z}^{(r)}\left(M^{k_{l}}\right)-\mathcal{Z}^{(r)}\left(M^{k_{l-1}}\right)\right]$ and $M^{0}=Z$ by definition. By assuming the existence of $\mathcal{V}_{\infty}$ and $\sigma_{\infty}$ such that $v\left(k_{l-1}, k_{l}\right)=\mathcal{V}_{\infty} / \sqrt{k_{l}}$ and $v\left(0, k_{0}\right)=\sigma_{\infty}$, at least for large enough $k_{0}$, we get

$$
\operatorname{Var}\left[Y^{\mathbf{k}, \mathbf{n}}\right]=\frac{\sigma_{\infty}^{2}}{n_{0}}+\sum_{l=1}^{L} n_{1}^{-1} \kappa^{l-1} \frac{\mathcal{V}_{\infty}^{2}}{k_{0} \kappa^{l}}=\frac{\sigma_{\infty}^{2}}{n_{0}}+\frac{k_{0}^{-1} \mathcal{V}_{\infty}^{2} L^{2}}{N \kappa^{L}-n_{0}}
$$


The minimization of the latter expression over $n_{0}$ gives

$$
n_{0}^{*}=\frac{N \kappa^{L}}{1+L \sqrt{\frac{\mathcal{V}_{\infty}^{2} \kappa^{L}}{\sigma_{\infty}^{2} K}}}=\frac{N \kappa^{L}}{1+L \sqrt{v^{2}\left(k_{L-1}, K\right) \kappa^{L} / \sigma_{\infty}^{2}}} .
$$

Hence

$$
n_{1}^{*}=n_{0}^{*} \sqrt{\frac{v^{2}\left(k_{L-1}, K\right) \kappa^{L-2}}{\sigma_{\infty}^{2}}}
$$

yielding

$$
\operatorname{Var}\left[Y^{\mathbf{k}, \mathbf{n}^{*}}\right]=\frac{1}{N \kappa^{L}}\left(\sigma_{\infty}+L \sqrt{v^{2}\left(k_{L-1}, K\right) \kappa^{L}}\right)^{2}=: \frac{\Theta_{K, L}^{2}}{N} .
$$

Since the variance of $Y^{K, N}$ is $\sigma_{\infty}^{2} / N$, we get

$$
\mathcal{R}(K, L):=\frac{\operatorname{Var}\left[Y^{\mathbf{k}, \mathbf{n}^{*}}\right]}{\operatorname{Var}\left[Y^{K, N}\right]}=\left(\kappa^{-L / 2}+\frac{L v\left(k_{L-1}, K\right)}{\sigma_{\infty}}\right)^{2} .
$$

In the numerical experiment in the next section, we take $k_{0}=K \kappa^{-L}$, and $n_{0}^{*}$ and $n_{1}^{*}$ according to formulas (21) and (22), respectively, for any fixed $K$ and $N$.

Discussion The above comparison method has the following key features.

- The comparison results do not depend on the actual bias and bias rate (hence complexity rate) of the $\mathrm{AB}$ algorithm.

- For fixed $K$, the variance ratio $\mathcal{R}(K, L)$ in (23) is independent of $N$ (hence $C$ ).

- For any $L, K^{\prime}>K$, it holds that $\mathcal{R}\left(K^{\prime}, L\right) \leq \mathcal{R}(K, L)$.

- If $K$ is sufficiently large, then there exists $L_{K}$ such that $\mathcal{R}\left(K, L_{K}\right)=\min _{L \geq 1} \mathcal{R}(K, L)<$ 1 (significantly).

These features may be interpreted as follows. Let $K$ be as in the last bullet point. Then every $\mathrm{AB}$ estimate due $K^{\prime}$ inner simulations, $K^{\prime} \geq K$, and $N$ outer simulations, involving a total computational costs of $C^{\prime}:=N K^{\prime}$, will be outperformed by a particular ML estimate at the same costs $C^{\prime}$, having the same bias but (significantly) smaller variance. While the ML accuracy gain based on this comparison strategy might not be very tremendous, the results are robust in the sense that comparison of an optimal ML parametrization with an optimal $\mathrm{AB}$ parametrization (which both depend on particular knowledge of the bias rate) is avoided. Put differently, with any given $K^{\prime}>K$ for the AB method, there will be some connected optimal $N$ that depends on the actual complexity of the $\mathrm{AB}$ algorithm for the problem under consideration. The above comparison then shows that whatever this $N$ may be, i.e., whatever budget $C^{\prime}=N K^{\prime}$ we choose for $\mathrm{AB}$, with this budget we may outperform the corresponding $\mathrm{AB}$ estimate with a more accurate ML estimate using an number of levels $L_{K}:=\min _{L \geq 1} \mathcal{R}(K, L)$. Furthermore, it should be noted that in a next turn the ML estimate might be improved further, since the parameter choices due to the above strategy may not be optimal. Indeed, we know that for large enough budgets the achievable ML accuracy is (asymptotically) of higher order than the $\mathrm{AB}$ accuracy, and so an ML parametrization that implies the AB bias must be (asymptotically) suboptimal. 


\subsection{Bermudan swaptions in the LIBOR market model}

As a case study, we here consider the Bermudan swaption example from Sec. 7 in Kolodko and Schoenmakers (2006) in the context of the well known LIBOR Market Model. This example is chosen as it is somehow representative for a situation where a natural but suboptimal stopping strategy can be constructed in a canonical way, and where the corresponding dual price is asked for.

Remark 12 Another interesting case study would be the (25 dimensional!) asset based cancelable snowball swap studied in Bender et al. (2008). The latter case is a typical example where the method of Longstaff and Schwartz (2001) fails to give a very tight lower bound, hence where one is faced with a suboptimal stopping rule that yields a significant duality gap. The description and study of this example is considered too extensive for the present context however, and therefore postponed to a possible future numerical study.

The dynamics of the LIBOR Market Model with respect to a tenor structure $0<T_{1}<\ldots<T_{n}$ in the spot LIBOR measure $P^{*}$. are given by the following system of SDE's (for more details see, e.g., Schoenmakers (2005) and the references therein),

$$
d L_{i}=\sum_{j=\kappa(t)}^{i} \frac{\delta_{j} L_{i} L_{j} \gamma_{i} \cdot \gamma_{j}}{1+\delta_{j} L_{j}} d t+L_{i} \gamma_{i} \cdot d W^{*} \quad 0 \leq t \leq T_{i}, \quad 1 \leq i<n,
$$

with $\delta_{i}:=T_{i+1}-T_{i}, t \rightarrow \gamma_{i}(t)=\left(\gamma_{i, 1}(t), \ldots, \gamma_{i, d}(t)\right)$ being deterministic factor loadings, and $\kappa(t):=\min \left\{m: T_{m} \geq t\right\}$ being the next LIBOR fixing date after $t$. In $(24),\left(W^{*}(t) \mid 0 \leq\right.$ $\left.t \leq T_{n-1}\right)$ is a $d$-dimensional standard Brownian motion under the measure $P^{*}$ induced by the numeraire

$$
B_{*}(t):=\frac{B_{\kappa(t)}(t)}{B_{1}(0)} \prod_{i=1}^{\kappa(t)-1}\left(1+\delta_{i} L_{i}\left(T_{i}\right)\right)
$$

with $B_{i}(t), t \leq T_{i}$, being zero coupon bonds with face value $\$ 1$ at their respective maturities $T_{i}$, $1 \leq i \leq n$.

A Bermudan swaption issued at $t=0$ gives the holder the right to exercise once a cash-flow

$$
S\left(T_{i}\right):=\left(\sum_{j=i}^{n-1} B_{j+1}\left(T_{i}\right) \delta_{j}\left(L_{j}\left(T_{i}\right)-\theta\right)\right)^{+},
$$

that is the positive part of the value of a swap contract with settlement dates $T_{i+1}, \ldots, T_{n}$ and strike $\theta$, at an exercise date out of the set $\left\{\mathcal{T}_{1}, \ldots, \mathcal{T}_{\mathcal{J}}\right\} \subset\left\{T_{1}, \ldots, T_{n}\right\}$ specified in the option contract. So, in the terminology of Section 2 the discounted cashflow process reads

$$
Z_{j}:=S_{\mathcal{T}_{j}} / B_{*}\left(\mathcal{T}_{j}\right), \quad j=1, \ldots, \mathcal{J} .
$$

Let us recap the data used in the numerical example in Kolodko and Schoenmakers (2006):

- A flat $10 \%$ initial LIBOR curve over a 40 period quarterly tenor structure, i.e. $n=41, \delta_{i}=0.25$, 
- $\left|\gamma_{i}(t)\right|=c g\left(T_{i}-t\right)$,

where $g(s)=g_{\infty}+\left(1-g_{\infty}+a s\right) e^{-b s}$, with $c=0.2, a=1.5, b=3.5, g_{\infty}=0.5$,

- $\gamma_{i}(t)=c g\left(T_{i}-t\right) e_{i}$ with unit vectors $e_{i}$ being such that

$\rho_{i j}:=e_{i}^{\top} e_{j}=\exp (-\varphi|i-j|), i, j=1, \ldots, n-1$, with $\varphi=0.0413$.

We here consider only ATM Bermudan swaptions (i.e., $\theta=10 \%$ ) and yearly exercise opportunities, hence $\mathcal{T}_{i}=T_{4 i}, i=1, \ldots, 10$. The $\operatorname{SDE}(24)$ is simulated via a log-Euler scheme using $\Delta t=\delta / 5$, hence the solution may be regardes as "practically exact." As a stopping rule it is considered

$$
\tau_{i}=\inf \left\{j: i \leq j \leq \mathcal{J}, \max _{p: j \leq p \leq \mathcal{J}} \mathrm{E}^{j} Z_{p} \leq Z_{j}\right\}, \quad i=0, \ldots, \mathcal{J} .
$$

where the (discounted) European options $\mathrm{E}^{j} Z_{p}$ are computed using the standard swaption approximation formula (see e.g. Schoenmakers (2005)) that has accuracy better than than $0.3 \%$ relative for this example.

Based on $\tau_{i}$ we can define the martingale differences:

$$
\Delta_{i}=\mathrm{E}_{\mathcal{F}_{i}}\left[Z_{\tau_{i}}\right]-\mathrm{E}_{\mathcal{F}_{i-1}}\left[Z_{\tau_{i}}\right], \quad i=1, \ldots, \mathcal{J},
$$

and then construct a target martingale $M$ with $M_{0}=0$ and

$$
M_{i}=\Delta_{1}+\ldots+\Delta_{i}, \quad i=1, \ldots, \mathcal{J} .
$$

In order to compute the differences $\Delta_{i}$ we need sub-simulations. Note that $\mathrm{E}_{\mathcal{F}_{i}}\left[Z_{\tau_{i}}\right]=\mathrm{E}_{\mathcal{F}_{i}}\left[Z_{\tau_{i+1}}\right]$ if $\tau_{i}>i$ and $\mathrm{E}_{\mathcal{F}_{i}}\left[Z_{\tau_{i}}\right]=Z_{i}$ otherwise. Denote by $\Delta_{i}^{k}$ a Monte Carlo estimate for $\Delta_{i}$ based on two sets of $k$ inner trajectories starting from $X_{i-1}$ and $X_{i}$, respectively. Set for any $k \in \mathbb{N}$

$$
M_{i}^{k}=\Delta_{1}^{k}+\ldots+\Delta_{i}^{k}, \quad i=1, \ldots, \mathcal{J} .
$$

\section{Results}

Let us write our ML estimator in the form:

$$
\begin{aligned}
Y^{\mathbf{k}, \mathbf{n}} & =\frac{1}{n_{0}} \sum_{r=1}^{n_{0}} \mathcal{Z}^{(r)}\left(M^{k_{0}}\right)+\sum_{l=1}^{L} \frac{1}{n_{l}} \sum_{r=1}^{n_{l}}\left(\mathcal{Z}^{(r)}\left(M^{k_{l}}\right)-\mathcal{Z}^{(r)}\left(M^{k_{l-1}}\right)\right) \\
& =: \frac{1}{n_{0}} \sum_{r=1}^{n_{0}} \xi_{0, k_{0}}(r)+\sum_{l=1}^{L} \frac{1}{n_{l}} \sum_{r=1}^{n_{l}} \xi_{k_{l-1}, k_{l}}(r),
\end{aligned}
$$

where one and the same set of $k_{l}$ inner trajectories is used to compute both $\mathcal{Z}\left(M^{k_{l}}\right)$ and $\mathcal{Z}\left(M^{k_{l-1}}\right)$. In the first step, we take $n_{0}=10000, k_{0}=50, \kappa=2, L=3, n_{l}=n_{0} \kappa^{-l}, l=0, \ldots, L$, and compute the estimates:

$$
\widehat{v}\left(k_{l-1}, k_{l}\right)=\sqrt{\frac{1}{n_{l}} \sum_{r=1}^{n_{l}}\left(\xi_{k_{l-1}, k_{l}}(r)-\bar{\xi}_{k_{l-1}, k_{l}}\right)^{2}}, \quad \widehat{\Theta}_{K, L}=\frac{\widehat{\sigma}_{\infty}}{\kappa^{L / 2}}+L \widehat{v}\left(k_{L-1}, K\right)
$$

with $\widehat{\sigma}_{\infty}=\widehat{v}(0,300)$. The estimated values of $v\left(k_{l-1}, k_{l}\right)$ and $\Theta_{K, L}$ are presented in Table 1 together with the estimated "optimal" ratios $n_{0}^{*} / N$ and $n_{1}^{*} / N$ based on $\widehat{\sigma}_{\infty}$ and $\widehat{v}\left(k_{L-1}, K\right)$. The 
resulting variance ratio functions $\mathcal{R}(K, L), L=1,2,3$, are shown in Figure 1. In the second step, we fix the computational budget $N K=10^{6}$ and then use the optimal values $n_{0}^{*}$ and $n_{1}^{*}$ to compute the multilevel estimate $Y^{\mathbf{k}, \mathbf{n}^{*}}$. For comparison we also give the AB values $Y^{K, N}$ and their standard deviations. The results are shown in Table 2 for different values of $K$.

Table 1: Estimates for $v\left(k_{l-1}, k_{l}\right), n_{l}^{*}$ and $\Theta_{K}, l=1, \ldots, 3$

\begin{tabular}{|r|r|r|r|}
\hline$l$ & $\left(k_{l-1}, k_{l}\right)$ & $\widehat{v}\left(k_{l-1}, k_{l}\right)$ & $n_{l}^{*} / N$ \\
\hline \hline 0 & $(0,50)$ & 0.006928 & 0.006803 \\
1 & $(50,100)$ & 0.002918 & 0.002199 \\
2 & $(100,200)$ & 0.002048 & 0.001100 \\
3 & $(200,400)$ & 0.001386 & 0.000550 \\
\hline & $K=400$ & $\widehat{\Theta}_{K}=0.006300$ & \\
\hline \hline 0 & $(0,100)$ & 0.006055 & 0.003993 \\
1 & $(100,200)$ & 0.002044 & 0.001001 \\
2 & $(200,400)$ & 0.001521 & 0.000501 \\
3 & $(400,800)$ & 0.001075 & 0.000250 \\
\hline & $K=800$ & $\widehat{\Theta}_{K}=0.005366$ & \\
\hline \hline 0 & $(0,150)$ & 0.006083 & 0.003127 \\
1 & $(150,300)$ & 0.001581 & 0.000590 \\
2 & $(300,600)$ & 0.001118 & 0.000295 \\
3 & $(600,1200)$ & 0.000809 & 0.000148 \\
\hline & $K=1200$ & $\widehat{\Theta}_{K}=0.004569$ & \\
\hline \hline 0 & $(0,200)$ & 0.005986 & 0.002416 \\
1 & $(200,400)$ & 0.001443 & 0.000431 \\
2 & $(400,800)$ & 0.001040 & 0.000215 \\
3 & $(800,1600)$ & 0.000764 & 0.000108 \\
\hline & $K=1600$ & $\widehat{\Theta}_{K}=0.004435$ & \\
\hline \hline 0 & $(0,250)$ & 0.005918 & 0.001995 \\
1 & $(250,500)$ & 0.001360 & 0.000334 \\
2 & $(500,1000)$ & 0.000971 & 0.000167 \\
3 & $(1000,2000)$ & 0.000718 & 0.000084 \\
\hline & $K=2000$ & $\widehat{\Theta}_{K}=0.004297$ & \\
\hline \hline 0 & $(0,300)$ & 0.005954 & 0.001809 \\
1 & $(300,600)$ & 0.001198 & 0.000254 \\
2 & $(600,1200)$ & 0.000822 & 0.000127 \\
3 & $(1200,2400)$ & 0.000602 & 0.000064 \\
\hline & $K=2400$ & $\widehat{\Theta}_{K}=0.003949$ & \\
\hline & & & \\
\hline
\end{tabular}




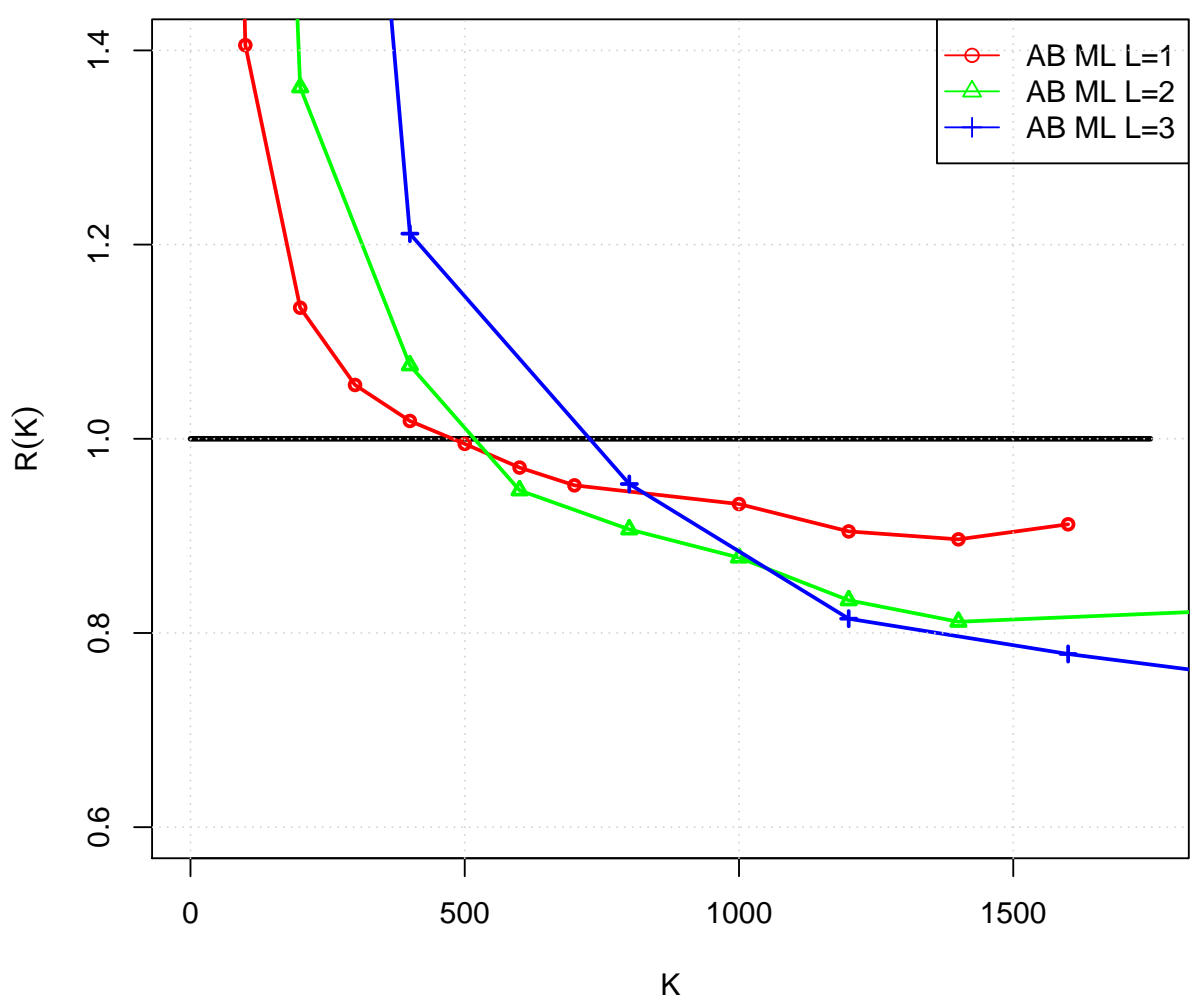

Figure 1: The variance ratio function $\mathcal{R}(K, L)$ for $L=1,2,3$. 


\section{Proofs}

\subsection{Proof of Theorem 5}

On the one hand, it holds for each $k \in \mathbb{N}$ and $j_{k}^{\max }:=\min \mathcal{Q}^{k}$ that

$$
\begin{aligned}
\mathcal{Z}\left(M^{k}\right)-\mathcal{Z}(M) & =\max _{j=0, \ldots, \mathcal{J}}\left(Z_{j}-M_{j}^{k}\right)-\max _{j=0, \ldots, \mathcal{J}}\left(Z_{j}-M_{j}\right) \\
& \leq M_{j_{k}^{\max }}-M_{j_{k}^{\max }}^{k}, \quad \text { a.s. },
\end{aligned}
$$

and on the other hand, we get for each $k \in \mathbb{N}$ and $\mathcal{Q}=:\left\{j^{\max }\right\}$,

$$
\mathcal{Z}\left(M^{k}\right)-\mathcal{Z}(M) \geq M_{j^{\max }}-M_{j^{\max }}^{k} .
$$

By (27) and (28) we thus have

$$
\mathrm{E}\left[\left(\mathcal{Z}\left(M^{k}\right)-\mathcal{Z}(M)\right)^{2}\right] \leq \mathrm{E}\left[\max _{j=1, \ldots, \mathcal{J}}\left(M_{j}-M_{j}^{k}\right)^{2}\right] \leq B k^{-\beta} .
$$

Further, by the Cauchy-Schwarz inequality we so have immediately,

$$
\left|\mathrm{E}\left[\mathcal{Z}\left(M^{k}\right)-\mathcal{Z}(M)\right]\right| \leq\left\{\mathrm{E}\left[\max _{j=1, \ldots, \mathcal{J}}\left(M_{j}-M_{j}^{k}\right)^{2}\right]\right\}^{1 / 2} \leq \sqrt{B} \cdot k^{-\beta / 2}
$$

Let us now turn to the case where in addition assumptions (AR'), (AL) and (AQ) are fulfilled. From (27) we obtain for $k \in \mathbb{N}$,

$$
\begin{aligned}
\mathrm{E}_{\mathcal{F}}\left[\mathcal{Z}\left(M^{k}\right)-\mathcal{Z}(M)\right] \leq & \mathrm{E}_{\mathcal{F}}\left[M_{j^{\max }}-M_{j_{k}^{\max }}^{k}\right] \\
= & \mathrm{E}_{\mathcal{F}}\left[\left(M_{j_{k}^{\max }}-M_{j_{k}^{\max }}^{k}+M_{j_{\max }}^{k}-M_{j^{\max }}\right) 1_{j_{k}^{\max } \neq j^{\max }}\right] \\
& +\mathrm{E}_{\mathcal{F}}\left[M_{j^{\max }}-M_{j^{\max }}^{k}\right]=:(I)+(I I) .
\end{aligned}
$$

Note that $Z_{j_{k}^{\max }}-M_{j_{k}^{\max }}^{k} \geq Z_{j^{\max }}-M_{j^{\max }}^{k}$ and hence

$$
\left\{j_{k}^{\max } \neq j^{\max }\right\} \subset\left\{\max _{j=1, \ldots, \mathcal{J}}\left(M_{j}-M_{j}^{k}+M_{j^{\max }}^{k}-M_{j^{\max }}\right) \geq \Lambda\right\} .
$$

We thus have

$$
\begin{aligned}
\mathrm{P}_{\mathcal{F}}\left(j_{k}^{\max } \neq j^{\max }\right) & \leq \mathrm{P}_{\mathcal{F}}\left(\max _{j=1, \ldots, \mathcal{J}}\left(M_{j}-M_{j}^{k}+M_{j^{\max }}^{k}-M_{j_{\max }} \geq \Lambda\right)\right. \\
& \leq \mathrm{P}_{\mathcal{F}}\left(\max _{j=1, \ldots, \mathcal{J}}\left(M_{j}-M_{j}^{k}\right) \geq \Lambda / 2\right)+\mathrm{P}_{\mathcal{F}}\left(M_{j_{\max }}^{k}-M_{j_{\max }} \geq \Lambda / 2\right) \\
& \leq \mathrm{P}_{\mathcal{F}}\left(\max _{j=1, \ldots, \mathcal{J}}\left(M_{j}-M_{j}^{k}\right) \geq \Lambda / 2\right)+\mathrm{P}_{\mathcal{F}}\left(\max _{j=1, \ldots, \mathcal{J}}\left(M_{j}^{k}-M_{j}\right) \geq \Lambda / 2\right)
\end{aligned}
$$

By (AR') and the conditional Markov inequality it follows that

$$
\mathrm{P}_{\mathcal{F}}\left(\max _{j=1, \ldots, \mathcal{J}}\left(M_{j}-M_{j}^{k}\right) \geq \Lambda / 2\right) \leq \frac{4 B}{\Lambda^{2}} k^{-\beta}, \quad \mathrm{P}_{\mathcal{F}}\left(\max _{j=1, \ldots, \mathcal{J}}\left(M_{j}^{k}-M_{j}\right) \geq \Lambda / 2\right) \leq \frac{4 B}{\Lambda^{2}} k^{-\beta} .
$$


Hence

$$
\mathrm{P}_{\mathcal{F}}\left(j_{k}^{\max } \neq j^{\max }\right) \leq \frac{8 B}{\Lambda^{2}} k^{-\beta}
$$

for all $k$. Furthermore, due to (AR'), and a conditional version of the generalized Hölder inequality

$$
E|X Y| \leq\|X\|_{p}\|Y\|_{q}
$$

for $\frac{1}{p}+\frac{1}{q} \leq 1$ and $p, q \geq 1$, we obtain by taking $q=2$ and $p=\max \{2,2 / a\}$,

$$
\begin{aligned}
(I) & \leq\left[\mathrm{P}_{\mathcal{F}}\left(j_{k}^{\max } \neq j^{\max }\right)\right]^{1 / p} \sqrt{4 \mathrm{E}_{\mathcal{F}}\left[\max _{j=1, \ldots, \mathcal{J}}\left(M_{j}-M_{j}^{k}\right)^{2}\right]} \\
& \leq \frac{2(8 B)^{1 / p}}{\Lambda^{2 / p}} k^{-\beta / p} B k^{-\beta / 2}=: \frac{B_{1}}{\Lambda^{2 / p}} k^{-\beta(1 / p+1 / 2)} .
\end{aligned}
$$

Combining (27) with (28) and using assumption (AR') again for the term (II), we arrive at the inequality

$$
-A k^{-\alpha} \leq \mathrm{E}\left[\mathcal{Z}\left(M^{k}\right)-\mathcal{Z}(M)\right] \leq B_{1} k^{-\beta(1 / p+1 / 2)} \mathrm{E}\left[\frac{1}{\Lambda^{2 / p}}\right]+A k^{-\alpha} \leq C k^{-\gamma}
$$

with $\gamma=\min \{\alpha, \beta \min \{1,(a+1) / 2\}\}$ and some $C>0$.

\subsection{Proof of Theorem 11}

Due to (9) and (11), we have in any case

$$
\left|\mathrm{E}\left[Y^{\mathbf{n}, \mathbf{k}}\right]-Y(M)\right| \leq C k_{0}^{-\gamma} \kappa^{-L \gamma}=\varepsilon / \sqrt{2}
$$

i) Case $\beta<1$ : We have by (10),

$$
\begin{aligned}
\operatorname{Var}\left[Y^{\mathbf{n}, \mathbf{k}}\right] & \leq \widetilde{B} \sum_{l=0}^{L} \widetilde{B}^{-1} 2^{-1} \varepsilon^{2} k_{0}^{\beta} \kappa^{-L(1-\beta) / 2}\left(1-\kappa^{-(1-\beta) / 2}\right) \kappa^{l(1+\beta) / 2} k_{0}^{-\beta} \kappa^{-\beta l} \\
& =2^{-1} \sum_{l=0}^{L} \varepsilon^{2} \kappa^{-L(1-\beta) / 2}\left(1-\kappa^{-(1-\beta) / 2}\right) \frac{\kappa^{(L+1)(1-\beta) / 2}-1}{\kappa^{(1-\beta) / 2}-1} \frac{\kappa^{-(1-\beta) / 2}}{\kappa^{-(1-\beta) / 2}} \\
& =2^{-1} \sum_{l=0}^{L} \varepsilon^{2} \kappa^{-L(1-\beta) / 2}\left(\kappa^{(L+1)(1-\beta) / 2}-1\right) \kappa^{-(1-\beta) / 2} \\
& =2^{-1} \varepsilon^{2}\left(1-\kappa^{-(L+1)(1-\beta) / 2}\right) \leq \varepsilon^{2} / 2,
\end{aligned}
$$


and (12) straightforwardly follows from (29). The order of $\mathcal{C}^{\mathbf{n}, \mathbf{k}}(\varepsilon)$ follows from the estimate

$$
\begin{aligned}
\sum_{l=0}^{L} k_{l} n_{l} & \leq \sum_{l=0}^{L} k_{0} \kappa^{l}\left(2 \varepsilon^{-2} \widetilde{B} k_{0}^{-\beta} \kappa^{L(1-\beta) / 2}\left(1-\kappa^{-(1-\beta) / 2}\right)^{-1} \kappa^{-l(1+\beta) / 2}+1\right) \\
& =\sum_{l=0}^{L} \kappa^{l}\left(2 \varepsilon^{-2} \widetilde{B} k_{0}^{1-\beta} \kappa^{L(1-\beta) / 2}\left(1-\kappa^{-(1-\beta) / 2}\right)^{-1} \kappa^{-l(1+\beta) / 2}+k_{0}\right) \\
& =k_{0}^{1-\beta}\left(2 \varepsilon^{-2} \widetilde{B} \kappa^{L(1-\beta) / 2}\left(\kappa^{L(1-\beta) / 2}-\kappa^{-(1-\beta) / 2}\right)+k_{0} \frac{\kappa^{L+1}-1}{\kappa-1}\right) \\
& \leq 2 \varepsilon^{-2} \widetilde{B} k_{0}^{1-\beta} \kappa^{L(1-\beta)}+k_{0} \kappa^{L+1} \\
& \leq 2 \varepsilon^{-2} \widetilde{B} k_{0}^{1-\beta} \kappa^{\left(\frac{-\ln \frac{k_{0}^{\gamma} \varepsilon}{C \sqrt{2}}}{\gamma \ln \kappa}\right)(1-\beta)}+k_{0} \kappa^{\frac{-\ln \frac{k_{0}^{\gamma} \varepsilon}{C \sqrt{2}}}{\gamma \ln \kappa}+2} \\
& =2 \widetilde{B} \frac{(C \sqrt{2})^{(1-\beta) / \gamma}}{\varepsilon^{2+(1-\beta) / \gamma}} \kappa^{(1-\beta)}+\frac{(C \sqrt{2})^{1 / \gamma}}{\varepsilon^{1 / \gamma}} \kappa^{2} \\
& =O\left(\varepsilon^{-2-(1-\beta) / \gamma}\right), \quad \varepsilon \rightarrow 0
\end{aligned}
$$

(note that $\gamma \geq 1 / 2$ ).

ii) Case $\beta=1$ : By a straightforward calculation, we obtain again

$$
\operatorname{Var}\left[Y^{\mathbf{n}, \mathbf{k}}\right] \leq \varepsilon^{2} / 2
$$

as well as (12) via (29). For $\mathcal{C}^{\mathbf{n}, \mathbf{k}}(\varepsilon)$ we now have

$$
\begin{aligned}
\mathcal{C}^{\mathbf{n}, \mathbf{k}}(\varepsilon) & \leq \sum_{l=0}^{L} k_{0} \kappa^{l}\left(2 \varepsilon^{-2} \widetilde{B}(L+1) k_{0}^{-1} \kappa^{-l}+1\right) \\
& =\sum_{l=0}^{L}\left(2 \varepsilon^{-2} \widetilde{B}(L+1)+k_{0} \kappa^{l}\right) \\
& =2 \varepsilon^{-2} \widetilde{B}(L+1)^{2}+k_{0} \frac{\kappa^{L+1}-1}{\kappa-1} \\
& \leq 2 \varepsilon^{-2} \widetilde{B}(L+1)^{2}+k_{0} \kappa^{L+1} \\
& \leq 2 \varepsilon^{-2} \widetilde{B}\left(\frac{-\ln \frac{k_{0}^{\gamma} \varepsilon}{C \sqrt{2}}}{\gamma \ln \kappa}+2\right)^{2}+\frac{(C \sqrt{2})^{1 / \gamma}}{\varepsilon^{1 / \gamma}} \kappa^{2} \\
& =O\left(\varepsilon^{-2} \ln ^{2} \varepsilon\right), \quad \varepsilon \rightarrow 0
\end{aligned}
$$

since $\gamma \geq 1 / 2$. 
iii) Case $\beta>1$ : For the variance we have

$$
\begin{aligned}
\operatorname{Var}\left[Y^{\mathbf{n}, \mathbf{k}}\right] & \leq \widetilde{B} \sum_{l=0}^{L} 2^{-1} \varepsilon^{2} \widetilde{B}^{-1} k_{0}^{\beta}\left(1-\kappa^{-(\beta-1) / 2}\right) \kappa^{l(1-\beta) / 2} k_{0}^{-\beta} \\
& =2^{-1} \varepsilon^{2}\left(1-\kappa^{-(\beta-1) / 2}\right) \frac{1-\kappa^{(L+1)(1-\beta) / 2}}{1-\kappa^{(1-\beta) / 2}} \\
& =2^{-1} \varepsilon^{2}\left(1-\kappa^{(L+1)(1-\beta) / 2}\right) \leq \varepsilon^{2} / 2
\end{aligned}
$$

and (12) holds in view of (29). Finally, we derive

$$
\begin{aligned}
\mathcal{C}^{\mathbf{n}, \mathbf{k}}(\varepsilon) & \leq \sum_{l=0}^{L} k_{0} \kappa^{l}\left(2 \varepsilon^{-2} \widetilde{B} k_{0}^{-\beta}\left(1-\kappa^{-(\beta-1) / 2}\right)^{-1} \kappa^{-l(1+\beta) / 2}+1\right) \\
& \leq 2 \varepsilon^{-2} \widetilde{B} k_{0}^{1-\beta} \sum_{l=0}^{L}\left(1-\kappa^{-(\beta-1) / 2}\right)^{-1} \kappa^{l(1-\beta) / 2}+k_{0} \kappa^{L+1} \\
& =2 \varepsilon^{-2} \widetilde{B} k_{0}^{1-\beta}\left(1-\kappa^{(L+1)(1-\beta) / 2}\right)+k_{0} \kappa^{L+1} \\
& \leq 2 \varepsilon^{-2} \widetilde{B} k_{0}^{1-\beta}+\frac{(C \sqrt{2})^{1 / \gamma}}{\varepsilon^{1 / \gamma}} \kappa^{2} \\
& =O\left(\varepsilon^{-2}\right), \quad \varepsilon \rightarrow 0,
\end{aligned}
$$

since $\gamma \geq 1 / 2$.

\section{References}

L. Andersen and M. Broadie (2004). A primal-dual simulation algorithm for pricing multidimensional American options. Management Sciences, 50(9), 1222-1234.

V. Bally, G. Pages (2003). A quantization algorithm for solving multidimensional discrete optimal stopping problem. Bernoulli, 9(6), 1003-1049.

D. Belomestny (2011). Pricing Bermudan options using regression: optimal rates of convergence for lower estimates. Finance and Stochastics, 15(4), 655-683..

D. Belomestny (2012). Solving optimal stopping problems by empirical dual optimization and penalization, to appear in Annals of Applied Probability.

D. Belomestny, C. Bender and J. Schoenmakers (2009). True upper bounds for Bermudan products via non-nested Monte Carlo. Math. Financ., 19(1), 53-71.

C. Bender, A. Kolodko, J. Schoenmakers (2008). Enhanced policy iteration for American options via scenario selection. Quant. Finance 8, No. 2, 135-146.

M. Broadie, P. Glasserman (2004). A stochastic mesh method for pricing high-dimensional American options. Journal of Computational Finance, 7(4), 35-72. 
M. Broadie and M. Cao (2008). Improved Lower and Upper Bound Algorithms for Pricing American Options by Simulation. Quantitative Finance, 8(8), 845-861.

J. Carriere (1996). Valuation of early-exercise price of options using simulations and nonparametric regression. Insurance: Mathematics and Economics, 19, 19-30.

M.H.A. Davis, I. Karatzas (1994). A Deterministic Approach to Optimal Stopping. In: Probability, Statistics and Optimisation (ed. F.P. Kelly). NewYork Chichester: John Wiley \& Sons Ltd., pp. 455-466

M.B. Giles (2008). Multilevel Monte Carlo path simulation. Operations Research 56(3):607-617.

P. Glasserman (2003). Monte Carlo Methods in Financial Engineering. Springer.

M. Joshi (2007). A simple derivation of and improvements to Jamshidian's and Rogers' upper bound methods for Bermudan options. Applied Mathematical Finance, 14, 197-205.

M. Haugh, L. Kogan (2004). Pricing American options: a duality approach. Operations Research, 52(2), 258-270.

M. Kohler, A. Krzyzak und H. Walk (2010). Upper bounds for Bermudan options on Markovian data using nonparametric regression and a reduced number of nested Monte Carlo steps. Statistics and Decision, 26, 275-288.

A. Kolodko, J. Schoenmakers (2004). Upper bounds for Bermudan style derivatives. Monte Carlo Methods and Appl., 10, No. 3-4, 331-343.

A. Kolodko, J. Schoenmakers (2006). Iterative construction of the optimal Bermudan stopping time. Finance and Stochastics, 10, 27-49.

F.A. Longstaff, E.S. Schwartz (2001). Valuing American options by simulation: a simple leastsquares approach. Review of Financial Studies, 14, 113-147.

L.C.G. Rogers (2002). Monte Carlo valuation of American options. Mathematical Finance, 12, 271-286.

J. Schoenmakers (2005), Robust Libor Modelling and Pricing of Derivative Products. BocaRaton London NewYork Singapore: Chapman \& Hall - CRC Press 2005

J. Schoenmakers, J. Huang, J. Zhang (2012). Optimal dual martingales, their analysis and application to new algorithms for Bermudan products. arXiv:1111.6038v2, earlier version by J. Schoenmakers, J. Huang (2011), http://dx.doi.org/10.2139/ssrn.1825944 SSRN.

J. Tsitsiklis, B. Van Roy (1999). Regression methods for pricing complex American style options. IEEE Trans. Neural. Net., 12, 694-703. 
Table 2: The performance of ML estimates with the optimal choice of $n_{l}^{*}, l=0, \ldots, 3$, compared to the performance of the standard $\mathrm{AB}$ estimate with $N K=10^{6}$ and $K=k_{L}$.

\begin{tabular}{|c|r|r|r|r|}
\hline$l$ & $n_{l}^{*}$ & $k_{l}$ & $\frac{1}{n_{l}} \sum_{r=1}^{n_{l}} \xi_{k_{l-1}, k_{l}}(r)$ & $\widehat{v}\left(k_{l-1}, k_{l}\right)$ \\
\hline \hline 0 & 6600 & 50 & 0.0341611 & 0.00686113 \\
1 & 2230 & 100 & $1.12787 \mathrm{e}-05$ & 0.00288741 \\
2 & 1110 & 200 & $2.45243 \mathrm{e}-05$ & 0.00193951 \\
3 & 550 & 400 & $2.74035 \mathrm{e}-05$ & 0.00140730 \\
\cline { 4 - 5 } & & & $Y^{\mathbf{n}^{*}, \mathbf{k}}=0.0340843$ & $\operatorname{sd}\left(Y^{\mathbf{n}^{*}, \mathbf{k}}\right)=\mathbf{0 . 0 0 0 1 3 3 6}$ \\
\hline \hline & & & & \\
$\mathrm{AB}$ & $N=2500$ & $K=400$ & $Y^{N, K}=0.0341167$ & $\operatorname{sd}\left(Y^{N, K}\right)=\mathbf{0 . 0 0 0 1 1 1 1}$ \\
\hline
\end{tabular}

\begin{tabular}{|c|r|r|r|r|}
\hline$l$ & $n_{l}^{*}$ & $k_{l}$ & $\frac{1}{n_{l}} \sum_{r=1}^{n_{l}} \xi_{k_{l-1}, k_{l}}(r)$ & $\widehat{v}\left(k_{l-1}, k_{l}\right)$ \\
\hline \hline 0 & 3880 & 100 & 0.0341174 & 0.00630161 \\
1 & 1010 & 200 & $6.64818 \mathrm{e}-06$ & 0.00208278 \\
2 & 500 & 400 & $4.17533 \mathrm{e}-05$ & 0.00144304 \\
3 & 250 & 800 & $6.16153 \mathrm{e}-05$ & 0.00101637 \\
\cline { 4 - 5 } & & & $Y^{\mathbf{n}^{*}, \mathbf{k}}=0.0340224$ & $\operatorname{sd}\left(Y^{\mathbf{n}^{*}, \mathbf{k}}\right)=\mathbf{0 . 0 0 0 1 5 1 0}$ \\
\hline \hline & & & & \\
$\mathrm{AB}$ & $N=1250$ & $K=800$ & $Y^{N, K}=0.0341235$ & $\operatorname{sd}\left(Y^{N, K}\right)=\mathbf{0 . 0 0 0 1 6 7 6}$ \\
\hline
\end{tabular}

\begin{tabular}{|c|r|r|r|r|}
\hline$l$ & $n_{l}^{*}$ & $k_{l}$ & $\frac{1}{n_{l}} \sum_{r=1}^{n_{l}} \xi_{k_{l-1}, k_{l}}(r)$ & $\widehat{v}\left(k_{l-1}, k_{l}\right)$ \\
\hline \hline 0 & 3050 & 150 & 0.034024 & 0.00613309 \\
1 & 600 & 300 & $5.96397 \mathrm{e}-06$ & 0.00167245 \\
2 & 300 & 600 & $-1.46135 \mathrm{e}-05$ & 0.00114155 \\
3 & 150 & 1200 & $1.42701 \mathrm{e}-05$ & 0.00078627 \\
\cline { 4 - 5 } & & & $Y^{\mathbf{n}^{*}, \mathbf{k}}=0.0340522$ & $\operatorname{sd}\left(Y^{\mathbf{n}^{*}, \mathbf{k}}\right)=\mathbf{0 . 0 0 0 1 5 9 5}$ \\
\hline \hline & & & & \\
$\mathrm{AB}$ & $N=850$ & $K=1200$ & $Y^{N, K}=0.0340616$ & $\operatorname{sd}\left(Y^{N, K}\right)=\mathbf{0 . 0 0 0 1 9 0 3}$ \\
\hline
\end{tabular}

\begin{tabular}{|c|r|r|r|r|}
\hline$l$ & $n_{l}^{*}$ & $k_{l}$ & $\frac{1}{n_{l}} \sum_{r=1}^{n_{l}} \xi_{k_{l-1}, k_{l}}(r)$ & $\widehat{v}\left(k_{l-1}, k_{l}\right)$ \\
\hline \hline 0 & 2360 & 200 & 0.0340537 & 0.00593753 \\
1 & 430 & 400 & $4.03549 \mathrm{e}-05$ & 0.00157739 \\
2 & 210 & 800 & $-7.94923 \mathrm{e}-06$ & 0.00095657 \\
3 & 100 & 1600 & $-7.50145 \mathrm{e}-05$ & 0.00090936 \\
\cline { 4 - 5 } & & & $Y^{\mathbf{n}^{*}, \mathbf{k}}=0.0340111$ & $\operatorname{sd}\left(Y^{\mathbf{n}^{*}, \mathbf{k}}\right)=\mathbf{0 . 0 0 0 1 8 2 6}$ \\
\hline \hline & & & & \\
$\mathrm{AB}$ & $N=625$ & $K=1600$ & $Y^{N, K}=0.0340312$ & $\operatorname{sd}\left(Y^{N, K}\right)=\mathbf{0 . 0 0 0 2 3 2 6}$ \\
\hline
\end{tabular}

